\title{
INFORMATION - STATISTICAL APPROACH TO INVERSE OPTICAL PROBLEM SOLUTION FOR 3D DISPERSE SYSTEMS WITH NANO- AND MICRO PARTICLES
}

\author{
ALEXANDRA G. BEZRUKOVA, OLGA L. VLASOVA \\ Peter the Great St. Petersburg Polytechnical University (St. Petersburg, Russian Federation)
}

Submitted 18 November 2021

(C) Belarusian State University of Informatics and Radioelectronics, 2021

\begin{abstract}
Multiparameter analysis of simultaneous optical data for $3 D$ disperse systems (consisted from nanoand/or microparticles of different nature) by information-statistical methods can help to estimate the share of different types of particles in mixtures. At the solution of inverse optical problem for unknown poly-component $3 D$ DS, the comparison of measured parameters with the known ones from the set of mono-component $3 D$ DS can help to identify the component content of the system under study. The approach was tested on the biomineral water mixtures of kaolin clay and bacterium coli bacillus with the help of the program based on the information-statistical theory. To solve the impurity optical recognition tasks, the Base of optical data for $3 D$ DS is needed.
\end{abstract}

Keywords: biomineral mixtures, $3 D$ disperse systems, information-statistical methods, micro and nanoparticles, online optical control.

Conflict of interests. The authors declare no conflict of interests.

Gratitude. The authors thank Dr. Dmitriy F. Kalinin for the opportunity to process experimental data using the program MultAlt.

For citation. Bezrukova A.G., Vlasova O.L. Information-statistical approach to inverse optical problem solution for $3 D$ disperse systems with nano- and micro particles. Doklady BGUIR. 2021; 19(8): 31-34.

\section{Introduction}

Ensembles of nano- and / or micro- particles can be considered as three-dimensional $(3 D)$ disperse systems (DS) with particles as a disperse phase in dispersive medium (water, air, etc.) [1]. The particle sizes of the $3 D$ DS studied at our work [2-7] vary from nanometers to approximately ten micrometers in an average diameter. This article is devoted to the application of information-statistical methods [8-11] to multiparameter optical analysis of bicomponents (BC) 3D DS such as biomineral mixtures of kaolin clay (polymodal $3 D$ DS consisted from nano and micro particles) and bacterium coli bacillus (with the average diameter about one micrometer) [5]. The inverse optical problem solution for polycomponent (PC) $3 D$ DS meets with difficulties due to the necessity of "a priori" information about component $3 D$ DS content. The set of parameters from optical methods: refractometry, absorbance, fluorescence, and light scattering (integral and differential, static and dynamic, unpolarized and polarized) is unique for each mono-component (MC) $3 D$ DS [6].

At the analysis of unknown PC $3 D$ DS, the comparison of measured parameters with the known ones for the set of MC $3 D$ DS can help to identify the system under study. For this purpose, the computer technology "MultAlt" [10] for practical prognosis based on the information-statistical 
theory [8-11] was used. This approach provides the process of interpretation and visualization of intermediate and final results with an estimation of decision probability.

\section{Methods of conducting experiment}

The information-statistical methodology was tested on experimental biomineral mixtures as a two component (BC) 3D DS consisted of kaolin clay [5] and bacterium coli bacillus (or Escherichia coli, or E. coli) of strain K-802 [5] in different proportions. Particle size distributions for both $3 D$ DS are polymodal with nano and micro particles [5].

In the previous articles [2-7] there were discussions about the main compatible optical methods for $3 D$ DS online characterization [7], polarization measurements information possibilities, and the $3 D$ DS polymodality [4]. $N D$ unique optical vector of $3 D$ DS $[6,7]$ is the $N D$ set of the so called "second-class" optical parameters by which the MC $3 D$ DS can be characterized and compared with other MC $3 D$ DS. One of the main second-class parameters is "wave exponent" $-n(\lambda)[1]$, which can be obtained by measuring on spectrophotometer the extinction of light due to the integral light scattering. At the interval of wavelength $(\lambda)$, where there is no absorbance of light by $3 D$ DS, optical density spectra $-D(\lambda)$, can be considered as a measure of the integral light scattering (except the aperture angle of a photo-receiver). On a bi-logarithmic scale the slope of the linear part of $D(\lambda)$ is $n(\lambda)$ (Fig. 1):

$$
n(\lambda)=-\Delta \lg D(\lambda) / \Delta \lg \lambda
$$

For the identification of potential objects in unknown $3 D$ DS mixtures the informationstatistical methodology of complex interpretation of experimental data [8-11] with the computer technology MultAlt [10] are considered.

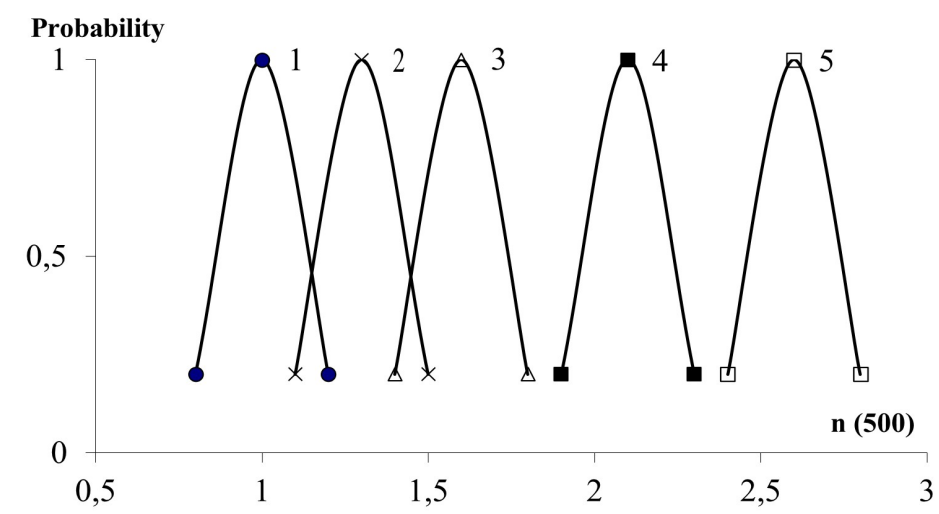

Fig. 1. The scheme of probability distributions for five experimental $3 D$ DS with the following $n(\lambda)$ values at $\lambda=500 \mathrm{~nm}: 1) 1.00$ by volume - only kaolin clay (MC $3 D \mathrm{DS}), n(500)=1.0 ; 2)$ mixture of 0.75 by volume kaolin clay and 0.25 - coli bacillus $(\mathrm{BC} 3 D \mathrm{DS}), n(500)=1.3 ; 3)$ mixture of 0.5 by volume - kaolin clay and 0.5 - coli bacillus (BC $3 D \mathrm{DS}), n(500)=1.6 ; 4)$ mixture of 0.25 by volume kaolin clay and $0.75-$ coli bacillus (BC $3 D$ DS), $n(500)=2.1 ; 5) 1.0$ by volume - only coli bacillus (MC $3 D$ DS $), n(500)=2,6$. Errors of $n(500)$ about 3-5\%

Earlier $[6,7]$ it was reported that a set of optical parameters of the so-called second class (obtained as a result of processing experimental data without involving any a priori information about $3 D$ DS) is unique for each $3 D$ DS and implicitly reflects characteristics of $3 D$ DS: shape, refractive index of particles, distribution functions of number and mass of particles in size, etc. In other words, the characteristic of any $3 D$ DS can be represented as an $N D$ vector in the $N$-dimensional space of optical parameters of the second class. On the basis of theory and experiment it is possible to predict specific parameters for a certain component. In the previous papers, the $3 D$ DS polymodality problem [4], optical characterization of $3 D$ DS mixtures [5], and the use of unique optical vectors for monitoring the aggregation process [7] were discussed. This part of study is connected with creation of algorithm based on the information-statistical methodology [8-11], which can help in the search of the most informative data for the particles of interest control (Fig. 1, 2). 


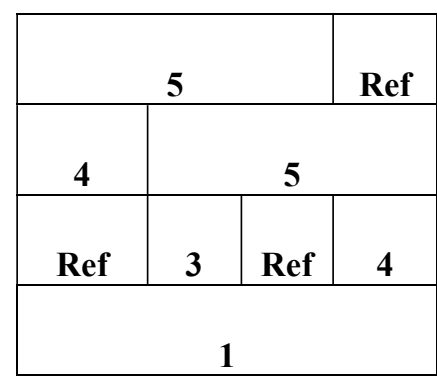

\begin{tabular}{|c|c|c|c|}
\hline \multicolumn{3}{|c|}{5} & Ref \\
\hline 4 & 4 & 4 & 5 \\
\hline 2 & 2 & \multicolumn{2}{|c|}{3} \\
\hline \multicolumn{4}{|c|}{1} \\
\hline \multicolumn{2}{|c|}{} \\
\hline
\end{tabular}

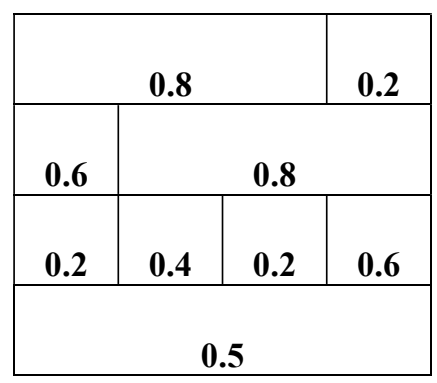

$a$

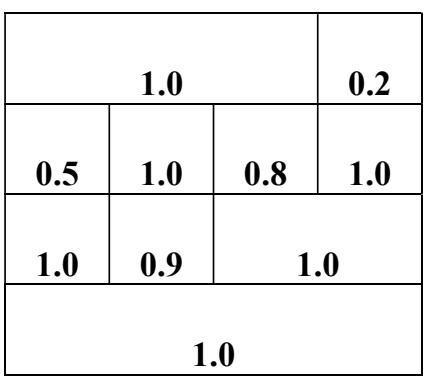

$b$

Fig. 2. MultAlt solutions (left) and probabilities of MultAlt solutions (right) based on the data from unique vectors for dispersions of kaolin clay (1), coli bacillus (5), and their mixtures $(2,3,4)$ are submitted in Fig. 1. The data based on one $(a)$ and five $(b)$ optical parameters of the second class. Note: mixture $2(a)$ did not appear at the analysis by this parameter of the second class. "Ref" means that the program MultAlt refused from solution with probability 0.2

\section{Experimental results and discussion}

The mixed dispersion of kaolin clay and bacterium coli bacillus can be designated as BC $3 D$ DS and considered as a model of natural water. With the help of a number of optical methods it is possible to obtain information on the distribution of particles in a multimodal $3 D$ DS [4]. However, the question of the component affiliation of different modes in mixtures remains open. In the study of unknown $3 D$ DS mixtures, there is a priori an uncertainty of knowledge about the type of the constituent particles, which requires the development of approaches to the interpretation of data. It seems that one of the promising areas in the study of various optical parameters, on the informative possibility to determine the component composition of unknown $3 D$ DS mixtures, is the method based on the information-statistical theory of optimal complexation [8-11].

The peculiarity of the input data is that instead of the component physical parameter, the probability of a component with this parameter presence is input (Fig. 1). The algorithms and programs developed on the base of optimal complexation can allow the analysis of PC $3 D$ DS by $N D$ vector optical parameters to identify the components the presence of which is the most probable. For example, the solution of five-alternative optical recognition task for mixtures of kaolin clay and bacterium coli bacillus by MultAlt program [10] is presented (Fig. 2).

\section{Conclusion}

In the unique vector approach after the detailed $3 D$ DS study there is a proposal of the optical parameter set which can be a useful tool for online analyzing complex $3 D$ DS. The $N D$ vector can characterize as unity the $3 D$ DS state with a minimum interference. For differentiation of $3 D$ DS constituents in mixtures the dimensions of $N D$ vectors can be enlarged due to the involvement into consideration of different measurement conditions such as wavelengths, angles and apertures of measurements, polarization, etc. Calculations are based only on the experimental data (without any models of particle structures and size distributions) and can be performed online. For better differentiation of the components in mixtures (for the solution of the impurity of optical recognition tasks for $3 D$ DS) the Base of optical data is needed $[2,3]$. 
Acknowledgements. Authors thank Prof. Vitaly J. Klenin, Prof. Sergei Ya. Frenkel, Prof. Feodor M. Goltsman, Prof. Alexander I. Melker, Dr. Tatyana B. Kalinina, Dr. Margarethe Hofmann, Dr Dmitriy F. Kalinin for the useful discussions. No external funding was received for this study.

\section{References}

1. Klenin V.J. Thermodynamics of systems containing flexible chain polymers. Elsevier; 1999.

2. Bezrukova A.G. Development of multiparametric optical assay for on-line environmental control. Proceedings of SPIE. 1997;3107:298-304.

3. Bezrukova A.G. Nondestructive testing of $3 D$ disperse systems with micro and nano particles: $N$-dimensional space of optical parameters. Proceedings of SPIE. 2006;6253:62530C1-C4.

4. Bezrukova A.G., Vlasova O.L. Optical characterization of $3 D$ disperse systems with nano and micro particles: polymodality of size distributions. Materials Physics and Mechanics. 2012;13(2):162-174.

5. Bezrukova A.G., Vlasova O.L. Mixtures of $3 D$ disperse systems with nano and micro particles: Optical Characterization. St. Petersburg Polytechnic University Journal, Physics and Mathematics. 2016;2(4):316-321. http://dx.doi.org/10.1016/j.spjpm.2016.11.004.

6. Bezrukova A.G., Vlasova O.L. Optical characterization of $3 D$ disperse systems with nano and micro particles: Unique vectors. Materials Physics and Mechanics. 2018;39(1):81-86. DOI: 10.18720/MPM.3912018_13.

7. Bezrukova A.G., Vlasova O.L. Nano and micro particle aggregation: unique optical vectors for control. Materials Physics and Mechanics. 2020;45(1):87-95. DOI: 10.18720/MPM.4512020_9.

8. Goltsman F.M. Physical experiment and statistical conclusions. Leningrad: Leningrad University Publishing House; 1982.

9. Kalinina T. B., Goltsman F.M. Methodological problems of interpretation of gravitational and magnetic Fields. Russian Geophysical Journal. 1994;(2):63-68.

10. Goltsman F.M., Kalinin D.F., Kalinina T.B. MULTALT computer technology for multi-alternative classification and forecasting for a complex of geodata. Russian Geophysical Journal. 2000;(17-18):64-70.

11. Kalinin D.F. Information and statistical prediction of mineral resources. St. Petersburg: Geological Exploration Group Publishing House; 2011.

\section{Authors' contribution}

Bezrukova A.G. and Vlasova O.L. - both authors performed the task for the study.

Bezrukova A.G. prepared the manuscript of the article.

\section{Information about the authors}

Bezrukova A.G., D.Sc., Senior Researcher, Corresponding member of St. Petersburg Academy of Sciences on Strength Problems, Peter the Great St. Petersburg Polytechnical University.

Vlasova O.L., D.Sc., Assistant Professor, Director of Graduate School of Biomedical Systems and Technologies in the Institute of Biomedical Systems and Biotechnology of Peter the Great St. Petersburg Polytechnical University.

\section{Address for correspondence}

195251, Russian Federation,

St. Petersburg, Polytekhnicheskaya St., 29.

Peter the Great St. Petersburg Polytechnical University;

e-mail: bezr@pb1097.spb.edu

Bezrukova Alexandra Gennadievna 